Pré-Publicações do Departamento de Matemática

Universidade de Coimbra

Preprint Number 09-21

\title{
SUBDIRECTLY IRREDUCIBLE SEPARABLE DYNAMIC ALGEBRAS
}

\author{
SANDRA MARQUES PINTO AND M. TERESA OLIVEIRA-MARTINS
}

\begin{abstract}
A characterization of the subdirectly irreducible separable dynamic algebras is presented. The notions developed for this study were also suitable to describe the previously found class of the simple separable dynamic algebras.

KEYWORDS: Boolean algebra with operators; dynamic algebra; dynamic congruence; essential element; subdirectly irreducible separable dynamic algebra; simple separable dynamic algebra.

AMS Subject Classification (1991): 03B05, 03B70, 03G05, 06E25.
\end{abstract}

\section{Introduction}

Both Rautenberg [Rau79] and Venema [Ven05] gave characterizations for subdirectly irreducible and simple Boolean algebras with operators. We have shown [Pin06] there are significant differences between congruences on dynamic algebras (pairs of a Boolean congruence and a regular congruence being closed to the heterogeneous operator (diamond),(cf. Definition 2.2)) and congruences on Boolean algebras with operators (Boolean congruences closed under operators). It is the propose of this work to show that results similar to those by Rautenberg and Venema can be achieved for the class of the separable dynamic algebras (Theorem 3.21 and Corollary 4.3).

\section{Dynamic algebras}

Dynamic algebras were introduced by Kozen [Koz79a] and Pratt [Pra79] to provide models for Propositional Dynamic Logic $(P D L)$. Following Pratt a dynamic algebra is a two-sorted algebra $(\mathcal{B}, \mathcal{R},\langle\rangle)$ verifying a certain set of equations. The Boolean universe (the universe of the propositions) $\mathcal{B}=$ $(B, \vee, \sim, 0)$ is a Boolean algebra (an algebra with a binary operation $\vee$, one unary operation $\sim$ and a nullary operation 0 , satisfying the usual set of axioms); the regular universe $\mathcal{R}=\left(R, \cup, ;,{ }^{*}\right.$ ) (or universe of actions or universe of programs) is an algebra with two binary operations, $\cup$,; and one

Received July 07, 2009.

Financial support from the Centro de Matemática da Universidade de Coimbra/FCT is gratefully acknowledged. 
unary operation *. The heterogeneous operator (diamond) $\langle\rangle:, R \times B \longrightarrow B$ relates both algebras.

Definition 2.1. A dynamic algebra $\mathcal{D}=\left(\mathcal{B}=(B, \vee, \sim, 0), \mathcal{R}=\left(R, \cup, ;{ }^{*}\right),\langle\rangle,\right)$ is an algebra satisfying

(1) $\mathcal{B}$ is a Boolean algebra

(2a) $\langle a, 0\rangle=0$

(2b) $\langle a, p \vee q\rangle=\langle a, p\rangle \vee\langle a, q\rangle$

(3) $\langle a \cup b, p\rangle=\langle a, p\rangle \vee\langle b, p\rangle$

(4) $\langle a ; b, p\rangle=\langle a,\langle b, p\rangle\rangle$

(5a) $p \vee\left\langle a ; a^{*}, p\right\rangle \leq\left\langle a^{*}, p\right\rangle$

(5b) $\left\langle a^{*}, p\right\rangle \leq p \vee\left\langle a^{*}, \sim p \wedge\langle a, p\rangle\right\rangle$

for $p, q \in B$ and $a, b \in R$.

For $p$ and $q$ elements of the Boolean algebra $\mathcal{B}$, we used $(p \leq q)$ as an abbreviation of $(p \vee q=q)$. Pratt [Pra79] showed that conditions (5a) and (5b) can be replaced by $\left\langle a^{*}, p\right\rangle=\min a ! p$ where $a ! p=\{q \in B: p \vee a q \leq$ $q\}$. For $a, b \in R$ and $p \in B$ we write $a p$ instead of $\langle a, p\rangle, a b$ instead of $a ; b$ and use $a \leq b$ whenever ( $a p \leq b p$, for every $p \in B$ ).

Let $\mathcal{D}=(\mathcal{B}, \mathcal{R},\langle\rangle)$ be a dynamic algebra and $a, b \in R$. We put $a \cong_{\mathcal{R}} b$, if $a p=b p$, for every proposition $p$ in $B$. The pair $\left(=, \cong_{\mathcal{R}}\right)$ defines a congruence relation ( $c f$. Definition 2.2, below) in the dynamic algebra $\mathcal{D}$. A dynamic algebra is separable if for any actions $a$ and $b$ in $R$, such that $a p=b p$, for any proposition $p \in B$, then $a=b$. For every dynamic algebra $\mathcal{D}$, the quotient algebra $\mathcal{D} /\left(=, \cong_{\mathcal{R}}\right)$ is separable.

The mixed operator diamond $\langle\rangle:, R \times B \longrightarrow B$ can be seen as

$$
\begin{aligned}
\langle\rangle: R & \longrightarrow F(B ; B) \\
a & \longrightarrow(p \longrightarrow\langle a\rangle p=\langle a, p\rangle)
\end{aligned}
$$

where $F(B ; B)=\{f \mid f: B \longrightarrow B$ is a mapping $\}$. However, unless $\mathcal{D}$ is separable, \langle\rangle does not define a representation of $R$ since \langle\rangle is not oneto-one. We may have equal mappings $\langle a\rangle$ and $\langle b\rangle$ in $F(B ; B)$ with $a \neq b$. Consequently, for $\mathcal{D}$ an arbitrary dynamic algebra, $\{\langle a\rangle: a \in R\}$ is a family of mappings in $B$ indexed by $R$ (where the extensionality axiom is not satisfied). In a separable dynamic algebra, $R$ may be identified with a set of mappings in $B$, that we will use whenever appropriate. Therefore, a dynamic algebra $\mathcal{D}$, can be viewed as a Boolean algebra with operators [Pra79]. Moreover, 
defining, for each $a \in R,[a] p:=\sim\langle a, \sim p\rangle$ for every $p \in B$, then $\left(\mathcal{B},\{[a]\}_{a \in R}\right)$ is a modal algebra ( $c f$. [Kra99]) associated with $\mathcal{D}$. The same modal algebra may be determined by distinct dynamic algebras.

The relation $\leq$ in $R, a \leq b$ whenever $(a p \leq b p$, for every $p \in B$ ) is reflexive and transitive, but it is not antisymmetric in general (since the conditions $a p \leq b p$ and $b p \leq a p$ give rise to $a p=b p$, for every $p \in B$ and not necessarily to $a=b$ ) and, therefore, defines a quasi-order in $R$. Clearly, in separable dynamic algebras $\leq$ defines a partial order since the antisymmetry is satisfied.

The elementary algebraic theory of dynamic algebras is similar to that of every other algebraic system [SB81], [Wec92]. We will specify some concepts for later use.

Definition 2.2. Let $\mathcal{D}=(\mathcal{B}, \mathcal{R},\langle\rangle)$ be a dynamic algebra. The pair $\theta=$ $\left(\theta_{1}, \theta_{2}\right)$ is a (dynamic) congruence relation on $\mathcal{D}$ if $\theta_{1}$ is a congruence relation on $\mathcal{B}, \theta_{2}$ is a congruence relation on $\mathcal{R}$ and $a p \theta_{1} b q$ whenever $\left(p \theta_{1} q\right.$ and $\left.a \theta_{2} b\right)$. We denote by $\operatorname{Cong}(\mathcal{D})$ the lattice of all dynamic congruence on $\mathcal{D}$.

In a dynamic algebra $\mathcal{D}=(\mathcal{B}, \mathcal{R},\langle\rangle)$ we define the congruences $\Delta_{B}$ and $\nabla_{B}$ on $B$ and $\Delta_{R}$ and $\nabla_{R}$ on $R$ as expected:

$$
\begin{array}{ll}
\Delta_{B}=\{(p, p): p \in B\} & \nabla_{B}=\{(p, q): p, q \in B\} \\
\Delta_{R}=\{(a, a): a \in R\} & \nabla_{R}=\{(a, b): a, b \in R\} .
\end{array}
$$

The pairs $\left(\Delta_{B}, \Delta_{R}\right),\left(\nabla_{B}, \nabla_{R}\right)$ and $\left(\nabla_{B}, \Delta_{R}\right)$ are congruences on $\mathcal{D}$, but, in general, $\left(\Delta_{B}, \nabla_{R}\right)$ is not a congruence on $\mathcal{D}$. Next we establish the conditions under which, for $\theta_{2}$ congruence on $\mathcal{R}$, the pair $\left(\Delta_{B}, \theta_{2}\right)$ is a dynamic congruence.

Proposition 2.3. Let $\mathcal{D}=(\mathcal{B}, \mathcal{R},\langle\rangle)$ be a separable dynamic algebra. Then $\left(\Delta_{B}, \theta_{2}\right)$ is a congruence if and only if $\theta_{2}=\Delta_{R}$.

Proof: If card $R=1$ then $\Delta_{R}$ is the unique existing regular congruence. Let us admit that card $R>1$. If $\left(\Delta_{B}, \theta_{2}\right)$ is a congruence and if $\theta_{2} \neq \Delta_{R}$, then there exist distinct elements $a, b \in R$ such that $a \theta_{2} b$. Immediately, $a p \Delta_{B} b p$, for each $p \in B$, i.e., $a p=b p$, for every $p \in B$. Since $\mathcal{D}$ is separable then $a=b$, a contradiction. Therefore $\theta_{2}=\Delta_{R}$. 
Corollary 2.4. On a separable dynamic algebra $\mathcal{D}=(\mathcal{B}, \mathcal{R},\langle\rangle)$ $\left(\Delta_{B}, \nabla_{R}\right)$ is a congruence iff card $R=1 \quad\left(\right.$ iff $\left.\nabla_{R}=\Delta_{R}\right)$.

\section{Subdirectly Irreducible Separable Dynamic Algebras}

Although every finite algebra is isomorphic to a direct product of directly indecomposable algebras, the same does not hold for infinite algebras. To solve this problem Birkhoff was led to consider subdirectly irreducible algebras.

For $\mathcal{A}$ arbitrary algebra we denote by $\mathcal{C}$ ong $(\mathcal{A})$ the lattice of all congruence on $\mathcal{A}$.

Definition 3.1. [DenWis02] An algebra $A$ is subdirectly irreducible if and only if $A$ is trivial or there is a minimum congruence in $\mathcal{C}$ ong $(\mathcal{A})-\{\Delta\}$ (if and only if there exist two elements $x, y$ in $A$ such that $x \theta y$, for any no trivial congruence $\theta$ ). In the latter case the minimum element is given by $\cap(\mathcal{C}$ ong $(\mathcal{A})-\{\Delta\})$ (a principal congruence).

The importance of the class of the subdirectly irreducible algebras is well stated on the following results due to Birkhoff.

Theorem 3.2. (Birkhoff) Every algebra $A$ is isomorphic to a subdirect product of subdirectly irreducible algebras (which are homomorphic images of A).

Theorem 3.3. (Birkhoff) Every finite algebra is isomorphic to a subdirect product of a finite number of subdirectly irreducible finite algebras.

The class of the simple algebras is a relevant subclass of the subdirectly irreducible algebras.

Definition 3.4. An algebra $A$ is simple if and only if $\operatorname{Cong}(\mathcal{A})=\{\Delta, \nabla\}$.

At [Pin06] the class of all simple separable dynamic algebras was described.

Definition 3.5. [Pin06] Let $\mathcal{D}=(\mathcal{B}, \mathcal{R},\langle\rangle)$ be a separable dynamic algebra with regular part containing an element $\exists_{s}$ satisfying $\exists_{s} 0=0$ and $\exists_{s} p=1$, for every $p \neq 0$. We call this element of $R$ the simple quantifier.

Theorem 3.6. [Pin08] The separable dynamic algebras $\left(\mathcal{B},\left\{\exists_{s}\right\},\langle\rangle\right)$ with $\mathcal{B}$ arbitrary are the unique simple separable dynamic algebras. 
Using [Pin06] we are able to conclude that a separable dynamic algebras, $\mathcal{D}=(\mathcal{B}, \mathcal{R},\langle\rangle)$, with Boolean part containing one or two elements is subdirecty irreducible. In fact we have

(1) If $B=\{0\}$ then $R=\{0\}$, and therefore, $\mathcal{D}$ is a degenerated dynamic algebra (admitting only one congruence).

(2) If $B=\{0,1\}$ then $R=\left\{i d_{R}\left(=\exists_{s}\right)\right\}$ or $R=\left\{\mathrm{o}, i d_{R}\right\}$ (where o $p=0$, for every $p \in B$ ).

(a) In the case $R=\left\{i d_{R}\left(=\exists_{s}\right)\right\}$ then $\left(\Delta_{B}, \Delta_{R}\right)$ and $\left(\nabla_{B}, \nabla_{R}\right)$ are the unique existing dynamic congruences and therefore $\mathcal{D}$ is simple and hence subdirectly irreducible.

(b) If $R=\left\{\mathrm{o}, i d_{R}\right\}$ then $\left(\Delta_{B}, \Delta_{R}\right),\left(\nabla_{B}, \nabla_{R}\right)$ and $\left(\nabla_{B}, \Delta_{R}\right)$ are the unique dynamic congruences. Since the minimum element of the set $\mathcal{C}$ ong $\mathcal{D}-\left\{\left(\Delta_{B}, \Delta_{R}\right)\right\}$ exists (equal to $\left.\left(\nabla_{B}, \Delta_{R}\right)\right)$ then $\mathcal{D}$ is subdirectly irreducible.

Therefore to obtain insight into the notion of subdirectly irreducibility we are led to study separable dynamic algebras

$$
\mathcal{D}=\left(\mathcal{B}=(B, \vee, \sim, 0), \mathcal{R}=\left(R, \cup, ;{ }^{*}\right),\langle,\rangle\right)
$$

with Boolean part having $2^{n}$ elements (i.e., $\operatorname{card} B=2^{n}$ ) for $n \in \mathbb{N}, n>1$.

As stated before, separable dynamic algebras can be seen as Boolean algebras with operators. But since the notions of dynamic congruences and modal congruences do not agree, a more detailed study of the former concept must be endured. (We note, for instance, that modal algebras are congruence distributive [Kra99] but separable dynamic algebras are not [Pin08].) On Boolean algebras with operators Venema [Ven05] used the notion of essential Boolean element to characterize the class of the subdirectly irreducible algebras. Now our aim is to establish, in the class of the separable dynamic algebras, an entity with a similar role to the one expressed by an essential element in Boolean algebras with operators.

Definition 3.7. Let $\mathcal{D}=(\mathcal{B}, \mathcal{R},\langle\rangle)$ be a separable dynamic algebra, $p_{e} \in B$ and $a \in R$. The pair $\left(p_{e}, a\right)$ is called an essential dynamic element in $\mathcal{D}$ if $p_{e} \neq 1$ and for every $p \neq 0$ there exists $b \in R$ satisfying

(1) $a=b^{*}$

(2) $b^{*} p \geq \sim p_{e}$. 
Proposition 3.8. Let $\mathcal{D}=(\mathcal{B}, \mathcal{R},\langle\rangle)$ be a separable dynamic algebra with regular part containing the simple quantifier $\exists_{s}$. For every $p \in B$, with $p \neq 1$, the pair $\left(p, \exists_{s}\right)$ is an essential dynamic element in $\mathcal{D}$.

Proof: Since $\exists_{s}^{*} q=\exists_{s} q=1$ for every $q \in B$ and $q \neq 0$, then $\exists_{s}^{*} q \geq \sim p$, for each $p \in B$, and therefore, for every $p \in B$, with $p \neq 1$, the pair $\left(p, \exists_{s}\right)$ is an essential dynamic element in $\mathcal{D}$.

We examine the existence of essential dynamic elements on two separable dynamic algebras.

Example 3.9. Let us consider the separable dynamic algebra $\mathcal{D}=(\mathcal{B}, \mathcal{R},\langle\rangle)$ where $B=\{0, p, q, 1\}, R=\{a\}$, with

$$
\begin{aligned}
& a 0=0 \\
& a p=p \\
& a q=1 \\
& a 1=1 .
\end{aligned}
$$

The structure $\mathcal{D}$ is, in fact, a dynamic algebra, since we have $a \cup a=a$,

$$
\begin{aligned}
& a a 0=a 0=0=a 0 \\
& a a p=a p=p=a p \\
& a a q=a 1=1=a q \\
& a a 1=a 1=1=a 1,
\end{aligned}
$$

so $a a=a$,

$$
\begin{aligned}
& a^{*} 0=\min \{s: 0 \vee a s \leq s\}=0=a 0 \\
& a^{*} p=\min \{s: p \vee a s \leq s\}=p=a p \\
& a^{*} q=\min \{s: q \vee a s \leq s\}=1=a q \\
& a^{*} 1=\min \{s: 1 \vee a s \leq s\}=1=a 1
\end{aligned}
$$

and therefore $a^{*}=a$.

The pairs $(0, a)$ and $(p, a)$ are not essential dynamic elements since, despite the fact that $a=a^{*}$, we have, respectively, $a p=p \nsupseteq \sim 0=1$ and $a p=p \nsupseteq$ $\sim p=q$.

The pair $(q, a)$ is an essential dynamic element, since

$$
\begin{aligned}
& a p=p \geq \sim q=p \\
& a q=1 \geq \sim q=p \\
& a 1=1 \geq \sim q=p .
\end{aligned}
$$


Example 3.10. The separable dynamic algebra $\mathcal{D}=(\mathcal{B}, \mathcal{R},\langle\rangle)$ with $B=$ $\{0, p, q, 1\}$ and $R=\{i d\}$ has no essential dynamic elements. The pairs $(0, i d),(p, i d)$ and $(q, i d)$ are not essential dynamic elements since we have, respectively, $i d(p) \nsupseteq \sim 0=1, i d(p) \nsupseteq \sim p=q$ and $i d(q) \nsupseteq \sim q=p$.

To obtain the characterization of the subdirectly irreducible separable dynamic algebras, new notions and results derived thereoff are required.

Definition 3.11. Let $\mathcal{D}=(\mathcal{B}, \mathcal{R},\langle\rangle)$ be a separable dynamic algebra. A subset $F$ of $B$ is a open Boolean filter of $\mathcal{D}$ if

(1) $F$ is a Boolean filter $\left(p_{1} \wedge p_{2} \in F\right.$, whenever $p_{1}, p_{2} \in F$; and $q \in F$ whenever $p_{1} \leq q$ and $\left.p_{1} \in F\right)$;

(2) $[a] p \in F$, for $a \in R$ and $p \in F$.

We denote by $\mathcal{F}_{o p}(\mathcal{D})$ the collection of all the open Boolean filters on $\mathcal{D}$.

Remark 3.12. In the above definition the condition $[a] p \in F$, whenever $a \in R$ and $p \in F$ is equivalent to $\left[a^{*}\right] p \in F$, whenever $a^{*} \in R$ and $p \in F$.

In fact, if $[a] p \in F$ for any $a \in R$, in particular, $\left[a^{*}\right] p \in F$ since $a^{*} \in R$. Conversely, using the fact that, for any $a \in R$, we have $a^{*} \geq a$, then $\left[a^{*}\right] p \leq$ $[a] p$, for every $p \in B$. So, if $\left[a^{*}\right] p \in F$ then $[a] p \in F$ (by definition of Boolean filter) as required.

Definition 3.13. Let $\mathcal{D}=(\mathcal{B}, \mathcal{R},\langle\rangle)$ be a separable dynamic algebra. A Boolean congruence $\theta$ is said to be a open Boolean congruence on $\mathcal{D}$ if

(1) $\theta$ is a Boolean congruence on $\mathcal{B}$;

(2) $a p \theta a q$ whenever $p \theta q$ and $a \in R$.

We denote by $\mathcal{C}_{o n g}(\mathcal{D})$ the collection of all the open Boolean congruences on $\mathcal{D}$.

Proposition 3.14. Let $\mathcal{D}=(\mathcal{B}, \mathcal{R},\langle\rangle)$ be a separable dynamic algebra. Then

(1) the collection of all open Boolean filters, $\mathcal{F}_{\text {op }}(\mathcal{D})$, is closed under taking arbitrary intersections and hence forms a lattice with respect to subset ordering;

(2) this lattice is isomorphic to the lattice of the open Boolean congruences on $\mathcal{D}, \mathcal{C}_{\text {ong }}(\mathcal{D})$, through the isomorphism

$$
\Pi: \mathcal{F}_{\text {op }}(\mathcal{D}) \longrightarrow \mathcal{C}_{\text {ong }} \text { op }(\mathcal{D})
$$

given by

$$
\Pi_{F}:=\{(p, q) \in B \times B: p \leftrightarrow q \in F\}
$$


and its inverse

$$
N: \mathcal{C}_{\text {ong }}(\mathcal{D}) \longrightarrow \mathcal{F}_{\text {op }}(\mathcal{D})
$$

by

$$
N_{\theta}:=\{p \in B: p \theta 1\} .
$$

Proof: (1) An immediate consequence of the definitions.

(2) Let us assume that $F$ is an open Boolean filter on $\mathcal{D}$. We pretend to prove that $\Pi_{F}:=\{(p, q) \in B \times B: p \leftrightarrow q \in F\}$ is an open Boolean congruence on $\mathcal{D}$. Using results on Boolean algebras we know that $\Pi_{F}$ is a Boolean congruence. It remains to be proved that, if $(p, q) \in \Pi_{F}$ then $(a p, a q) \in \Pi_{F}$, for every $a \in R$. But, if $(p, q) \in \Pi_{F}$ then, by definition of $\Pi, p \leftrightarrow q \in F$. From $p \rightarrow q \in F$ we get $[a](p \rightarrow q) \in F$ since $F$ is an open filter. Being the implication $[a](p \rightarrow q) \rightarrow([a] p \rightarrow[a] q)$ a K. Segerberg' axiom for PDL [Seg77], and since both $[a](p \rightarrow q)$ and $[a](p \rightarrow q) \rightarrow([a] p \rightarrow[a] q)$ are elements in $F$ then $[a] p \rightarrow[a] q \in F$. Similarly $[a] q \rightarrow[a] p \in F$. Hence $[a] p \leftrightarrow[a] q \in F$ as required.

Assuming that $\theta$ is an open Boolean congruence on $\mathcal{D}$ we intend to prove that $N_{\theta}:=\{p \in B: p \theta 1\}$ is an open Boolean filter on $\mathcal{D}$. It is immediate that $N_{\theta}$ is a Boolean filter. It remains to be proved that, if $p \in N_{\theta}$ then $[a] p \in N_{\theta}$, for every $a \in R$. If $p \in N_{\theta}$ then $p \theta 1$, so $\sim p \theta 0$, and since $\theta$ is an open congruence, for every $a \in R$, we have $a(\sim p) \theta a 0$, i.e., $a(\sim p) \theta 0$. Therefore $\sim a(\sim p) \theta 1$, and $\sim a(\sim p) \in N_{\theta}$, yielding the required $[a] p \in N_{\theta}$.

Proposition 3.15. Let $\mathcal{D}=(\mathcal{B}, \mathcal{R},\langle\rangle)$ be a separable dynamic algebra and $s$ any element of $B$. The set

$$
\begin{gathered}
F_{s}=\left\{q \in B:\left[a^{*}\right] s<q, \text { for some } a^{*} \in R\right\} \\
\left(\text { or } F_{s}=\left\{q \in B: a^{*}(\sim s) \geq \sim q, \text { for some } a^{*} \in R\right\}\right)
\end{gathered}
$$

is the smallest open Boolean filter on $\mathcal{D}$ containing s.

Proof: First we notice that $F_{s}$ is a Boolean filter. In fact, for $p, q \in F_{s}$ there exist elements $a^{*}$ and $b^{*}$ of $R$ such that

$$
a^{*}(\sim s) \geq \sim p, \text { and } b^{*}(\sim s) \geq \sim q .
$$


Therefore

$$
a^{*}(\sim s) \vee b^{*}(\sim s) \geq \sim p \vee \sim q,
$$

i.e.,

$$
\left(a^{*} \cup b^{*}\right)(\sim s) \geq \sim(p \wedge q) .
$$

But $\left(a^{*} \cup b^{*}\right)^{*}(\sim s) \geq\left(a^{*} \cup b^{*}\right)(\sim s)$ so there exists $c^{*}\left(=\left(a^{*} \cup b^{*}\right)^{*}\right) \in R$ such that

$$
c^{*}(\sim s) \geq \sim(p \wedge q) .
$$

Then $p \wedge q \in F_{s}$.

Let $p \in F_{s}$ and $q \in B$ such that $p \leq q$. Then there exists $a^{*} \in R$ such that

$$
\left[a^{*}\right] s<p .
$$

But since $p \leq q$ then

$$
\left[a^{*}\right] s<q,
$$

and therefore $q \in F_{s}$.

We have just proved that $F_{s}$ is a Boolean filter.

Now it is to be proved that for $p \in F_{s}$ and $a \in R$ then $[a] p \in F_{s}$.

Since $p \in F_{s}$ there exists $b^{*} \in R$ such that

$$
b^{*}(\sim s) \geq \sim p
$$

hence

$$
a b^{*}(\sim s) \geq a(\sim p) .
$$

¿From

$$
\left(a b^{*}\right)^{*}(\sim s) \geq a b^{*}(\sim s)
$$

we get

$$
\left(a b^{*}\right)^{*}(\sim s) \geq a(\sim p) .
$$

Therefore

$$
\left(a b^{*}\right)^{*}(\sim s) \geq \sim(\sim a(\sim p)),
$$

i.e., there exists $c^{*}\left(=\left(a b^{*}\right)^{*}\right) \in R$ such that

$$
c^{*}(\sim s) \geq \sim([a] p),
$$

and so $[a] p \in F_{s}$.

The proof that $s \in F_{s}$ is an immediate consequence of the fact

$$
a^{*} p \geq p
$$

for any $a^{*} \in R$ and any $p \in B$. Putting $p:=\sim s$ we have

$$
a^{*}(\sim s) \geq \sim s .
$$


Therefore $s \in F_{s}$.

It remains to be proved that $F_{s}$ is the smallest filter containing $s$.

Let $F$ be a filter containing $s$. We intend to show that $F_{s} \subseteq F$. For $p \in F_{s}$ there exists $a^{*} \in R$ such that $\left[a^{*}\right] s<p$. Since $s \in F$, an open Boolean filter of $\mathcal{D}$, and $a^{*} \in R$ then $\left[a^{*}\right] s \in F$ so $p \in F$.

Proposition 3.16. Let $\mathcal{D}=(\mathcal{B}, \mathcal{R},\langle\rangle)$ be a separable dynamic algebra. The set $F_{p_{e}}$ is the smallest nontrivial open Boolean filter on $D$ if and only if the pair $\left(p_{e}, a^{*}\right)$ is an essential element on $\mathcal{D}$ for some $a^{*} \in R$.

Proof: If $F$ is the smallest nontrivial open Boolean filter on $\mathcal{D}$ necessarily $F=F_{p_{e}}$ for some $p_{e}$ on $\mathcal{D}$ with $p_{e} \neq 1$. We intend to prove that there exists $a^{*} \in R$ such that $\left(p_{e}, a^{*}\right)$ is an essential element on $\mathcal{D}$. Let $q<1$ and let us consider the smallest open Boolean filter on $\mathcal{D}$ containing $q, F_{q}$. Since $F_{p_{e}}$ is the smallest nontrivial open Boolean filter on $\mathcal{D}$ then $F_{p_{e}} \subseteq F_{q}$, and therefore $p_{e} \in F_{q}$. So there exists $a^{*} \in R$ such that $\left[a^{*}\right] q \leq p_{e}$, i.e., $a^{*}(\sim q) \geq \sim p_{e}$, for $q \neq 1$, so $a^{*} p \geq \sim p_{e}$, for $p \neq 0$, and so $\left(p_{e}, a^{*}\right)$ is an essential element on $\mathcal{D}$.

Now let us admit that there exists an $a^{*} \in R$ such that $\left(p_{e}, a^{*}\right)$ is an essential element on $\mathcal{D}$. Let $F$ be a nontrivial open Boolean filter on $\mathcal{D}$. Since $F$ is nontrivial then $F \neq\{1\}$, so there exists $p \in F, p \neq 1$ (or $\sim p \neq 0$ ). But since $\left(p_{e}, a^{*}\right)$ is essential, then $a^{*}(\sim p) \geq \sim p_{e}$ and so $\left[a^{*}\right] p<p_{e}$. The fact that $p \in F$ implies $\left[a^{*}\right] p \in F$ and so $p_{e} \in F$. Therefore $F_{p_{e}} \subseteq F$ as required.

A Boolean congruence $\theta_{1}$ is called dynamical on a separable dynamic algebra $\mathcal{D}=(\mathcal{B}, \mathcal{R},\langle\rangle$,$) whenever there exists a congruence \theta_{2}$ on $\mathcal{R}$ such that $\left(\theta_{1}, \theta_{2}\right)$ is a congruence on $\mathcal{D}$. Examples of non dynamical congruences can be found in [Pin08].

Proposition 3.17. [Pin08] Let $\theta_{1}$ be a Boolean congruence on $\mathcal{D}$, a separable dynamic algebra. Then $\theta_{1}$ is dynamical on $\mathcal{D}$ if and only if $\left(\theta_{1}, \Delta_{\mathcal{R}}\right)$ is a dynamic congruence on $\mathcal{D}$.

Proposition 3.18. [Pin08] If $\theta_{1}$ is a dynamical congruence on $\mathcal{D}=(\mathcal{B}, \mathcal{R},\langle\rangle)$, a separable dynamic algebra, then $\left(\theta_{1}, \Delta_{\mathcal{R}}\right)$ is the smallest dynamic congruence on $\mathcal{D}$ having $\theta_{1}$ as Boolean part.

The following statement shows that the notion of open Boolean congruence on a separable dynamic algebra, $\mathcal{D}$, is equivalent to the concept of dynamical congruence on $\mathcal{D}$ given in [Pin08]. 
Proposition 3.19. On a separable dynamic algebra $\mathcal{D}=(\mathcal{B}, \mathcal{R},\langle\rangle)$ a congruence $\theta_{1} \in \mathcal{C} \operatorname{cng}_{\text {op }}(\mathcal{D})$ if and only if the pair $\left(\theta_{1}, \Delta_{R}\right) \in \mathcal{C}$ ong $(\mathcal{D})$ (if and only if $\theta_{1}$ is a dynamical congruence on $\mathcal{D}$ ).

Proof: Let $\theta_{1} \in \mathcal{C}_{\operatorname{ong}}(\mathcal{D})$. Then, for every $a \in R$, if $p \theta_{1} q$ then $a p \theta_{1} a q$, an so $\left(\theta_{1}, \Delta_{R}\right)$ is a dynamic congruence on $\mathcal{D}$. The converse is similar.

The existence of the minimum element on the set $\operatorname{Cong}(\mathcal{D})-\left\{\left(\Delta_{B}, \Delta_{R}\right)\right\}$ is obtained by the existence of the minimum element on $\mathcal{C}$ ong $g_{\text {op }}(\mathcal{D})-\left\{\Delta_{B}\right\}$, a fundamental result to be used in the proof of our main assertion, Theorem 3.21 .

Proposition 3.20. Let $\mathcal{D}=(\mathcal{B}, \mathcal{R},\langle\rangle)$ be a separable dynamic algebra. The minimum element on $\mathcal{C}$ ong $(\mathcal{D})-\left\{\left(\Delta_{B}, \Delta_{R}\right)\right\}$ exists if and only if there exists the minimum element on $\mathcal{C}$ ong ${ }_{\text {op }}(\mathcal{D})-\left\{\Delta_{B}\right\}$.

Proof: If the minimum element on $\mathcal{C}$ ong $(\mathcal{D})-\left\{\left(\Delta_{B}, \Delta_{R}\right)\right\}$ exists, using Proposition 3.18, this minimum is of the form $\left(\theta_{1}, \Delta_{R}\right)$ for some Boolean congruence $\theta_{1}$. Being $\left(\theta_{1}, \Delta_{R}\right)$ a dynamic congruence on $D$, then $\theta_{1}$ is an open Boolean congruence $D$ and so $\theta_{1} \subseteq \gamma_{1}$ for every Boolean congruence $\gamma_{1}$ on $B$ such that $\left(\gamma_{1}, \Delta_{R}\right)$ is a dynamic congruence on $\mathcal{D}$, i.e., $\theta_{1} \subseteq \gamma_{1}$, for every open Boolean congruence $\gamma_{1}$ on $\mathcal{D}$. So $\theta_{1}$ is the minimum element on $\mathcal{C}_{\text {ong }}(\mathcal{D})-\left\{\Delta_{B}\right\}$. And conversely.

As in Boolean algebras with operators [Ven05], the existence on a separable dynamic algebra of a unique Boolean element (associated with a essential dynamic element) determines the subdirectly irreducible dynamic algebras.

Theorem 3.21. A separable dynamic algebra $\mathcal{D}=(\mathcal{B}, \mathcal{R},\langle\rangle)$ is subdirectly irreducible if and only if there exists a unique element $p_{e} \in B$ such that the pair $\left(p_{e}, a^{*}\right)$ is an essential element on $D$, for some $a^{*} \in R$.

Proof: By Definition 3.1, a separable dynamic algebra is subdirectly irreducible if and only if the set $\operatorname{Cong}(D)-\left\{\left(\Delta_{B}, \Delta_{R}\right)\right\}$ has a minimum element. Attending to Proposition 3.20 this minimum exists if and only if there exists a minimum on the set $\mathcal{C}_{\text {ong }}$ op $(\mathcal{D})-\left\{\Delta_{B}\right\}$. But attending to Proposition 3.14, the existence of this minimum is equivalent to the existence of the smallest open filter on $\mathcal{D}$ of the type $F_{p_{e}}$. Now Proposition 3.16 implies that this filter is the smallest open filter on $\mathcal{D}$ if the pair $\left(p_{e}, a^{*}\right)$ is an essential element on $\mathcal{D}$ for some $a^{*} \in R$. 
We illustrate the result established by Theorem 3.21 with the following examples.

Example 3.22. Previously at Example 3.9 we presented a separable dynamic algebra $\mathcal{D}=(\mathcal{B}, \mathcal{R},\langle\rangle)$ with $B=\{0, p, q, 1\}, R=\{a\}$, where

$$
\begin{aligned}
& a 0=0 \\
& a p=p \\
& a q=1 \\
& a 1=1 .
\end{aligned}
$$

containing a unique essential dynamic element, the pair $(q, a)$. Therefore by Proposition 3.21 this dynamic algebra is subdirectly irreducible. In fact, $\mathcal{C}$ ong $\mathcal{R}=\left\{\Delta_{R}\right\}$ and $\mathcal{C}$ ong $\mathcal{B}=\left\{\Delta_{B}, \theta_{1}, \gamma_{1}, \nabla_{B}\right\}$ where

$$
\theta_{1}=\left\{\Delta_{B},(0, p),(p, 0),(q, 1),(1, q)\right\}
$$

and

$$
\gamma_{1}=\left\{\Delta_{B},(0, q),(q, 0),(p, 1),(1, p)\right\} .
$$

The pair $\left(\theta_{1}, \Delta_{R}\right)$ is a (dynamic) congruence since

$$
\begin{array}{llll}
0 \theta_{1} 0 \text { implies } & a 0 \theta_{1} a 0 & \text { so } & 0 \theta_{1} 0 \\
0 \theta_{1} p \text { implies } & a 0 \theta_{1} a p & \text { so } & 0 \theta_{1} p \\
1 \theta_{1} q \text { implies } & a 1 \theta_{1} a q & \text { so } & 1 \theta_{1} 1 \\
1 \theta_{1} 1 \text { implies } & a 1 \theta_{1} a 1 & \text { so } 1 \theta_{1} 1 .
\end{array}
$$

Nevertheless the pair $\left(\gamma_{1}, \Delta_{R}\right)$ is not a (dynamic) congruence since $0 \gamma_{1} q$ but $a 0 \not_{1} a q$ (i.e. $\left.0 \not_{1} 1\right)$.

Therefore

$$
\mathcal{C o n g} \mathcal{D}=\left\{\left(\Delta_{B}, \Delta_{R}\right),\left(\theta_{1}, \Delta_{R}\right),\left(\nabla_{B}, \nabla_{R}\left(=\Delta_{R}\right)\right)\right\} .
$$

The minimum element of the set $\mathcal{C}$ ong $\mathcal{D}-\left\{\left(\Delta_{B}, \Delta_{R}\right)\right\}$ exists (and is equal to $\left.\left(\theta_{1}, \Delta_{R}\right)\right)$. Therefore $\mathcal{D}$ is subdirectly irreducible.

Example 3.23. At Example 3.10 the separable dynamic algebra $\mathcal{D}=(\mathcal{B}, \mathcal{R},\langle\rangle)$ with $B=\{0, p, q, 1\}$ and $R=\{i d\}$ contains no dynamic essential elements and so is a non-subdirectly irreducible dynamic algebra.

In fact, since $\mathcal{C}$ ong $\mathcal{R}=\left\{\Delta_{R}\right\}$ and $\mathcal{C}$ ong $\mathcal{B}=\left\{\Delta_{B}, \theta_{1}, \gamma_{1}, \nabla_{B}\right\}$ with

$$
\theta_{1}=\left\{\Delta_{B},(0, p),(p, 0),(q, 1),(1, q)\right\}
$$

and

$$
\gamma_{1}=\left\{\Delta_{B},(0, q),(q, 0),(p, 1),(1, p)\right\}
$$


we have

$$
\mathcal{C} \text { ong } \mathcal{D}=\left\{\left(\Delta_{B}, \Delta_{R}\right),\left(\theta_{1}, \Delta_{R}\right),\left(\gamma_{1}, \Delta_{R}\right),\left(\nabla_{B}, \nabla_{R}\left(=\Delta_{R}\right)\right)\right\} .
$$

Since the minimum element of the set $\mathcal{C}$ ong $\mathcal{D}-\left\{\left(\Delta_{B}, \Delta_{R}\right)\right\}$ does not exist, $\mathcal{D}$ is not subdirectly irreducible.

\section{Simple Separable Dynamic Algebras}

Similarly to Boolean algebras with operators, the simple separable dynamic algebras are fully characterized by its essential elements.

Proposition 4.1. Let $\mathcal{D}=(\mathcal{B}, \mathcal{R},\langle\rangle)$ be a separable dynamic algebra. If for some $a \in R$ the pair $(0, a)$ is an essential dynamic element on $\mathcal{D}$ then $a=\exists_{s}$. Proof: By definition we have $b^{*} p \geq \sim 0=1$ for some $b \in R$ (with $b^{*}=a$ ) for any $p \neq 0$. Immediately $b^{*}=\exists_{s}$.

Theorem 4.2. A separable dynamic algebra $\mathcal{D}=(\mathcal{B}, \mathcal{R},\langle\rangle)$ is simple if and only if, for every $a \in R$, the pair $(0, a)$ is an essential dynamic element.

Proof: Let $\mathcal{D}=(\mathcal{B}, \mathcal{R},\langle\rangle)$ be a simple separable dynamic algebra. Then by Theorem 3.6 the algebra $\mathcal{D}$ is of the form $\mathcal{D}=\left(\mathcal{B},\left\{\exists_{s}\right\},\langle\rangle\right)$ for any Boolean algebra $\mathcal{B}$. Immediately, by Proposition 3.8, the pair $\left(0, \exists_{s}\right)$ is an essential dynamic element.

Conversely, let us admit that for every $b \in R$, the pair $(0, b)$ is an essential dynamic element. By Proposition 4.1 we have $b=\exists_{s}$. Therefore $\mathcal{D}=$ $\left(\mathcal{B},\left\{\exists_{s 1}\right\},\langle\rangle\right)$ with $\mathcal{B}$ an arbitrary Boolean algebra, as pretended.

A corresponding result to the one found by Rautenberg for Boolean algebras with operators can now be asserted.

Corollary 4.3. A separable dynamic algebra $\mathcal{D}=(\mathcal{B}, \mathcal{R},\langle\rangle)$ is simple if and only if, for every element $p_{e} \in B, p_{e} \neq 1$ and $a \in R$, the pair $\left(p_{e}, a\right)$ is an essential dynamic element.

\section{References}

[DenWis02] K.Denecke, S.L. Wismath. Universal Algebra and Applications in the Theorethical computer Science, Chapman \& Hall/CRC

[Har79] D. Harel. First-Order Dynamic Logic, Lecture Notes in Computer Science, Vol. 68, Spriger Verlag, New York, 1979

[Kra99] M. Kracht. Tools and Techniques in Modal Logic, Number 142 in Studies in Logic. Elsevier, Amsterdam, 1999 
[Koz79a] D. Kozen. A representation theorem for models of ${ }^{*}$-free pdl, Report RC7864, IBM Research Center, Yorktown Heights, New York, Sept, 1979

[Pin06] S. Marques Pinto, M. Teresa Oliveira-Martins and M. Céu Pinto. Monadic Dynamic Algebras, Mathematical Logic Quarterly 52 (2) (2006), 134-150

[Pin08] S. Marques Pinto, M. Teresa Oliveira-Martins and M. Céu Pinto. Congruences on Dynamic Algebras, Logic Jnl IGPL 16 (1) (2008), 15-31

[Pra79] V.R. Pratt. Dynamic Algebras: Examples, Constructions, Applications, Technical Report MIT/Laboratory for Comp. Sci/TM-138, July 1979, pages 1-33, 1979, and Stud. Log. 50 3/4, 571-605, 1992

[Rau79] W. Rautenberg. Klassiche und nichtlassische Aussagenlogik, Vieweg and Sohn, 1979

[SB81] H. Sankappanavar and S. Burris. A Course in Universal Algebra, Springer-Verlag New York Heidelberg Berlin, 1981

[Seg77] K. Segerberg. A completeness Theorem in the modal logic of programs, Notices of the AMS, 24(6):A-522, 1977

[Ven02] Y. Venema. Duals of subdirectly irreducible modal algebras ILLC Publications, 2002

[Ven05] Y. Venema. Algebras and Coalgebras, preprint for the Handbook for Modal Logic (to appear)

[Wec92] W. Wechler. Universal Algebra for Computer Scientists, Springer, Berlin, 1992

[Wee89] M. Weese. Decidable extensions of the theory of Boolean algebras, Handbook of Boolean Algebras, Volume 3, Elsevier Science Publishers, 1989

Sandra Marques Pinto

CMUC, Department of Mathematics, University of Coimbra, 3001-454 Coimbra, Portugal

E-mail address: sandra@mat.uc.pt

M. Teresa Oliveira-Martins

CMUC, Department of Mathematics, University of Coimbra, 3001-454 Coimbra, Portugal

E-mail address: meresa@mat.uc.pt 